\title{
Representación de enfermedad y depresión en pacientes con ERCT y asma: comparación por enfermedad
}

\section{Illness representation and depression in patients with ESRD and asthma: A comparison by illness}

\author{
Isaias Vicente Lugo González, Yuma Yoaly Pérez Bautista, \\ Silvia Susana Robles Montijo y Cynthia Zaira Vega Valero ${ }^{I}$
}

Citación: Lugo G., I.V., Pérez B., Y.Y., Robles M., S.S. y Vega V., C.Z. (2019). Representación de enfermedad y depresión en pacientes con ERCT y asma: comparación por enfermedad. Psicología y Salud, 29(2), 237-247.

\begin{abstract}
RESUMEN
La severidad de la enfermedad debe considerar la perspectiva biomédica y la forma en que los pacientes perciben su padecimiento, es decir, la representación que tengan de su enfermedad, para comprender el desarrollo de repercusiones emocionales tales como los síntomas de depresión. El objetivo de este trabajo fue evaluar, comparar y relacionar la representación de la enfermedad, la percepción de su severidad y los síntomas de depresión en personas con enfermedad renal crónica terminal en hemodiálisis y pacientes con asma. Al efecto, se llevó a cabo un estudio observacional, correlacional y de alcance descriptivo-comparativo en el que participaron 90 personas con dichos padecimientos. Los resultados encontrados sugieren que más de la mitad de la muestra reportó síntomas de depresión. Se identificaron correlaciones positivas significativas entre la percepción de severidad de la enfermedad, la representación emocional y los síntomas de depresión. No se observaron diferencias estadísticamente significativas en las variables evaluadas al hacer la comparación por enfermedad. Se concluye que la forma de percibir la enfermedad puede ser un factor relevante para determinar su severidad y sus consecuencias emocionales.
\end{abstract}

Palabras clave: Representación de enfermedad; Percepción de severidad de enfermedad; Síntomas de depresión; Enfermedad renal crónica terminal; Asma.

\begin{abstract}
Examining the severity of diseases should include both the biomedical perspective and the way patients perceive their suffering. Such individual representation should help understand the development of emotional repercussions such as depression symptoms. The purpose of the present study was to assess, compare and relate illness representation, perceived illness severity and symptoms of depression in people with either end-stage renal disease in hemodialysis or asthma. An observational, correlational and descriptive-comparative study was carried out with 90 participants, 45 with ESRD and 45 with asthma. Results suggest that more than $50 \%$ of all participants report depressive symptoms, and there are significant positive correlations between perceived illness severity, emotional representation and symptoms of depression. Finally, when comparing by illness, no statistically significant differences were observed in the variables evaluated. It is concluded that the way illness is perceived can be a relevant factor to determine its severity and its emotional consequences.
\end{abstract}

Key words: Illness representation; Perception of illness severity; Depression symptoms; End stage renal disease; Asthma.

\footnotetext{
${ }^{1}$ Facultad de Estudios Superiores Iztacala, Universidad Nacional Autónoma de México, Av. De los Barrios s/n, Los Reyes Iztacala, 54090 Tlalnepantla, Edo. de México, México, tels. (55)56-23-12-93 y (55)56-23-12-94, correos electrónicos: isaiasvlg@comunidad.unam.mx, yoalyyu@comunidad.unam.mx, susana@unam.mx y czaira.vega@unam.mx. Artículo recibido el 28 de junio y aceptado el 25 de septiembre de 2018.
} 


\section{INTRODUCCIÓN}

$\mathrm{L}$ as enfermedades crónicas son padecimientos de larga duración y carecen de cura, lo que implica que deban utilizarse tratamientos sintomatológicos y que haya un ajuste constante a las demandas de la enfermedad (Barley y Lawson, 2016; Bravo, 2014; Grau, 2016; Hill-Briggs, 2003; Larsen, 2009).

Vivir con una enfermedad crónica conlleva la mayoría de las veces el desarrollo de alteraciones emocionales, como la depresión (Gerontoukou, Michaelidoy, Rekleiti, Saridi y Souliotis, 2015; Katon, 2011; Livneh y Antonak, 2005; Livneh, Lott y Antonak, 2004). Los síntomas depresivos se han investigado ampliamente en personas que sufren la llamada enfermedad renal crónica terminal (ERCT en lo sucesivo) y el asma debido a que la depresión es una de las comorbilidades psicológicas más prevalentes y guarda una estrecha relación con el control de la enfermedad, la calidad de vida, la adherencia a los tratamientos y las limitaciones funcionales, entre otros (Christensen y Ehlers, 2002; Di Marco et al., 2010; Foster, Lavoie y Bouler, 2011; González et al., 2011; Jansen, Rijken, Heijmans, Kaptein y Groenewegen, 2012; McKercher et al., 2013; Mucsi, 2008; Van Lieshout y MacQueen, 2008; Yorke, Fleming y Shuldham, 2007).

Se sabe que entre 30 y $80 \%$ de las personas con ERCT en hemodiálisis presentan algún grado de depresión (Cukor et al., 2007; Goh y Griva, 2018; Ibrahim, Desa y Chiew-Tong, 2011). Por ejemplo, McKercher et al. (2013) realizaron un estudio con 50 pacientes, encontrando que $26.5 \%$ de ellos mostraba síntomas de depresión ( $10 \%$ con depresión mayor); Moreno et al. (2004) evaluaron a 75 pacientes, identificando a $44 \%$ con síntomas de depresión ( $29.3 \%$ leve, $12 \%$ moderada y $2.7 \%$ severa), y Esquivel et al. (2009), trabajando con 54 pacientes, hallaron que $18.2 \%$ de ellos manifestaba síntomas de depresión leve, $20 \%$ moderada y $14.5 \%$ severa.

Por su parte, entre 1 y $48 \%$ de los pacientes con asma exhiben síntomas de depresión (Ciprandi, Schiavetti, Rindone y Ricciardolo, 2015; Deshmukh, Toelle, Usherwood, O'Grady y Jenkins, 2008; Di Marco et al., 2010; Espinosa et al., 2006; Opolski y Wilson, 2005; Ritz, Meuret, Trueba,
Fritzsche y von Leupoldt, 2013; Strine, Mokdad, Balluz, Berry y Gonzalez, 2008). Coban y Aydemir (2014) evaluaron a 174 pacientes asmáticos, encontrando que casi la mitad de ellos mostraba síntomas depresivos. Ryu, Chun, Lee y Chang (2010) valoraron a 227 pacientes con diferentes padecimientos respiratorios, mostrando sus resultados que $41 \%$ de los 37 pacientes con asma reportó síntomas moderados y severos de depresión. Di Marco et al. (2010) encontraron que 27\% de 315 pacientes manifestaba dicha síntomatología depresiva.

Los síntomas de depresión se encuentran ligados a la severidad, complejidad e implicaciones de la enfermedad (tipo de tratamiento, frecuencia de uso de medicamentos, forma de administración de los mismos y demandas propias de la enfermedad) (Bravo, 2014; DiMatteo, 2004; Grau, 2016; Larsen, 2009; Livneh y Antonak, 2004). En este sentido, la ERCT y el asma comparten características de cronicidad, pero sus causas, evolución y forma de tratamiento son diferentes. Por ejemplo, la ERCT es un padecimiento multicausado que implica la pérdida de la función renal. Las personas que la sufren dependen cada vez más, de forma permanente, de un tratamiento sustitutivo, como por ejemplo sesiones de hemodiálisis de tres horas de duración tres veces a la semana, restricción del consumo de líquidos y alimentos y uso de medicamentos complementarios.

Las principales causas de esta enfermedad son la nefropatía diabética, la hipertensión arterial y las enfermedades glomerulares (Skorecki, Green y Breenner, 2001). A su vez, el asma es una enfermedad respiratoria multicausada que se caracteriza por la inflamación crónica de las vías aéreas, obstrucción del flujo aéreo y sensibilidad e hiperreactividad a diversos estimulos. Las formas de tratamiento implican con frecuencia el uso de inhaladores para el control de la inflamación bronquial (al menos dos veces al día), inhaladores de rescate para la reducción de los síntomas durante su exacerbación, evitación de estímulos desencadenantes y uso de medicamentos orales (esteroides, antileocutrienos, inmunomoduladores o anticuerpos monoclonales) (Global Initiative for Asthma, 2016). Se desconocen claramente las causas de la enfermedad, pero se han hallado ciertos factores de riesgo asociados a su desarrollo, como atopia, 
historia familiar de asma y exposición a diferentes virus (Choo, Hoyte y Katial, 2014).

Tomando en cuenta estas descripciones de los padecimientos, es posible concluir que hay diferencias en su severidad, al menos en lo que respecta a su descripción clínica y a las demandas del tratamiento; sin embargo, según Horne y Weinman (2002), dicha severidad debe considerar la forma en que las personas perciben su padecimiento, es decir, la representación de su enfermedad y no solo su descripción biomédica. Tal propuesta se afianza en el hecho de que la severidad de la enfermedad definida solamente en términos biomédicos no necesariamente se vincula con las importantes alteraciones emocionales que trae consigo (Guzman y Nicassio, 2003; Thuné-Boyle, Myers y Newman, 2006); pese a ello, una percepción negativa de la enfermedad se asocia claramente con una sintomatología depresiva (Bahçecioğlu y Çevikakyl, 2014; Ibrahim et al., 2011; Lin, Chen, Hsieh y Chang, 2013; Lugo, Reynoso y Fernández, 2014; Rees, Chilcot, Donnellan y Soulsby, 2018).

\section{El modelo MSCRE}

Bajo el anterior esquema, según el llamado "modelo de sentido común y representación de la enfermedad", o MSCRE (Diefenbach y Leventhal, 1996), es posible estudiar la representación de la enfermedad y la percepción de su severidad; en el caso de esta última, su análisis corresponde a la evaluación de la temporalidad con la que una persona cree que la tendrá, así como a sus consecuencias percibidas (Diefenbach y Leventhal, 1996; Horne y Weinman, 2002).

El MSCRE surge formalmente en la década de los sesenta basado en estudios de mensajes informativos, miedo y conductas preventivas de salud. El objetivo de esos estudios era identificar las condiciones bajo las cuales una serie de mensajes sobre una amenaza a la salud inducían cambios en las actitudes y conductas de prevención. El MSCRE fue el resultado del desarrollo de un modelo previo llamado "de procesos paralelos", y actualmente se le considera parte de los modelos de autorregulación debido a que está organizado en tres etapas: 1) etapa perceptual, en la que se incluye el conjunto de creencias sobre la enfermedad (representación cognitiva y representación emocional), lo que implica que cada persona organiza, explica y describe la información perceptiva (síntomas), conceptual (diagnóstico o nombre de la enfermedad) y emocional (reacciones emocionales) que tiene ante su padecimiento; 2) etapa de respuesta, que implica poner en marcha las conductas de afrontamiento que, según las creencias de cada persona, se consideren necesarias para dar sentido a la enfermedad, y manejar las demandas y las reacciones emocionales que genera la misma, y 3) etapa de resultados, en la que se evalúan la funcionalidad de las conductas implementadas en el manejo de la enfermedad y las consecuencias emocionales, ya que dicha evaluación funge como un proceso de retroalimentación constante para determinar el empleo futuro de las conductas y una posible modificación (positiva o negativa) de la representación de enfermedad (Diefenbach y Leventhal, 1996; Kaptein et al., 2008; Petrie, Jago y Devcich, 2007). A pesar de la estructura original del modelo, una tendencia de la investigación es evaluar la etapa perceptual del modelo y víncular el contenido de la misma con diversas variables de la enfermedad o de sus repercusiones emocionales (Kaptein et al., 2008; Kaptein, Klok, Moss-Morris y Brand, 2010).

A partir de este contexto, la representación de la enfermedad está constituida por diversas subdimensiones: identidad, temporalidad, causas, consecuencias, control, coherencia y representación emocional, las cuales están basadas en la información de la que se dispone, el reporte de otros enfermos, los medios de comunicación, las experiencias que han sido significativas durante el proceso de enfermedad o anteriores a ella, y los propios síntomas. Por lo tanto, dependiendo de la representación así conformada, cada persona organizará su comportamiento y responderá emocionalmente de una forma particular (Diefenbach y Leventhal, 1996; Mora y McAndrew, 2013; Moss-Morris et al., 2002).

Las subdimensiones del MSCRE se pueden entender mejor de la siguiente manera: la identidad hace referencia a la experiencia perceptual de la enfermedad, es decir, el tipo, lugar y cantidad de síntomas o sensaciones somáticas que se consideren correspondientes a la enfermedad; la temporalidad se refiere a la duración que se cree 
que tendrá la enfermedad (aguda, crónica o cíclica); las causas tienen que ver con las creencias sobre lo que causó la enfermedad y que se pueden estudiar en términos de los agentes externos (virus, bacterias, estrés cotidiano), la susceptibilidad (edad, genética o herencia) y los comportamientos (consumo de alcohol, tabaco y estilo de vida); las consecuencias aluden a los efectos percibidos y experimentados en diferentes áreas (funcionamiento físico, psicológico y social y repercusiones económicas); el control remite a las creencias sobre la capacidad percibida de controlar la enfermedad y a las expectativas de un tratamiento médico que controle la enfermedad o que disminuya los síntomas experimentados (control del tratamiento); la coherencia se relaciona con la claridad con la que se entiende la enfermedad (tipo de síntomas, duración de los mismos y temporalidad de la enfermedad), y por último la representación emocional implica las reacciones emocionales derivadas de los síntomas de la enfermedad, las sensaciones somáticas experimentadas, o bien el resultado de vivir con un padecimiento crónico (Cameron y Moss-Morris, 2004; Diefenbach y Leventhal, 1996; Mora y McAndrew, 2013).

Finalmente, cabe resaltar que el MSCRE se ha utilizado para evaluar la representación de la enfermedad y su relación con diversas consecuencias emocionales en la ERCT (Jansen et al., 2013; Jansen et al., 2012) y el asma (Coban y Aydemir, 2014; Halm, Mora y Leventhal, 2006; Kaptein et al., 2008; Kaptein et al., 2010; Unni y Shiyanbola, 2016; Walker y Chen, 2010). Por lo tanto, derivado de las consideraciones anteriores, este trabajo tuvo como propósito evaluar, comparar y relacionar la representación de enfermedad, la percepción de su severidad y los síntomas de depresión en personas con ERCT y con asma.

\section{MÉTODO}

\section{Participantes}

Considerando un muestreo no probabilístico de sujetos voluntarios (Hernández, Fernández y Baptista, 2014), se llevó a cabo un estudio observacional, correlacional y de alcance descriptivo-comparativo (Méndez, Namihira, Moreno y Sosa, 2001) en el que participaron 90 personas con ERCT $(n=45)$ y asma $(n=45)$, atendidas en dos instituciones de salud especializadas en esos padecimientos de la Ciudad de México. La Tabla 1 muestra las características demográficas y clínicas de la muestra.

Tabla 1. Características demográficas y clínicas de los participantes con ERCT y asma.

\begin{tabular}{|c|c|c|c|c|c|}
\hline \multicolumn{3}{|c|}{$\operatorname{Asma}(N=45)$} & \multicolumn{3}{|c|}{ ERCT $(N=45)$} \\
\hline Variables & Frec. & $\%$ & Variables & Frec. & $\%$ \\
\hline \multicolumn{6}{|c|}{ Sexo } \\
\hline Mujeres & 32 & 71.1 & Mujeres & 29 & 64.4 \\
\hline Hombres & 13 & 28.9 & Hombres & 16 & 35.6 \\
\hline \multicolumn{6}{|c|}{ Estado civil } \\
\hline Casado & 19 & 42.2 & Casado & 21 & 46.7 \\
\hline Soltero & 15 & 33.3 & Soltero & 15 & 33.3 \\
\hline Divorciado & 5 & 11.1 & Divorciado & 6 & 13.3 \\
\hline Unión libre & 5 & 11.1 & Unión libre & 1 & 2.2 \\
\hline \multicolumn{6}{|c|}{ Escolaridad } \\
\hline Primaria & 11 & 24.4 & Primaria & 9 & 20 \\
\hline Secundaria & 11 & 24.4 & Secundaria & 12 & 26.7 \\
\hline Preparatoria & 9 & 20 & Preparatoria & 9 & 20 \\
\hline Técnica & & & Técnica & 9 & 20 \\
\hline Licenciatura & 9 & 20 & Licenciatura & 4 & 8.9 \\
\hline Ninguna & 3 & 6.7 & Ninguna & 1 & 2.2 \\
\hline Posgrado & 2 & 4.4 & Posgrado & 1 & 2.2 \\
\hline \multicolumn{6}{|c|}{ Ocupación } \\
\hline Hogar & 14 & 31.1 & Hogar & 17 & 37.8 \\
\hline Trabaja & 26 & 57.8 & Trabaja & 18 & 39.9 \\
\hline Estudiante & 5 & 11.1 & Estudiante & 3 & 6.7 \\
\hline Nada & & & Nada & 7 & 15.5 \\
\hline
\end{tabular}

\section{Instrumentos}

Se utilizaron los siguientes instrumentos:

Cedula de identificación. Esta cédula incluyó preguntas sobre los datos sociodemográficos de los participantes y los clínicos propios de la enfermedad.

Cuestionario Breve de Percepción de Enfermedad (B-IPQ) (Broadbent, Petrie, Main y Weinman, 2006). Dicho instrumento, destinado a evaluar la representación cognitiva y emocional de la enfermedad en formato breve, está constituido por nueve ítems: 1) consecuencias, 2) temporalidad, 3) control personal de la enfermedad, 4) control del tratamien- 
to, 5) identidad, 6) preocupación por la enfermedad, 7) comprensión de la enfermedad (coherencia), 8) representación emocional de la enfermedad y 9) causas de la enfermedad (pregunta abierta). Cada ítem, con excepción del 9, se responde en una escala continua de once puntos que van de 0 a 10 . Cuenta con los siguientes datos de confiabilidad (test-retest): consecuencias $(\mathrm{r}=.70)$, temporalidad $(\mathrm{r}=.67)$, control personal $(\mathrm{r}=.63)$, control del tratamiento $(\mathrm{r}=.55)$, identidad $(\mathrm{r}=.55)$, preocupación $(\mathrm{r}=.66)$, coherencia $(\mathrm{r}=.48)$ y representación emocional $(\mathrm{r}=.65)$. Ejemplos de los ítems son, a saber: "¿En qué medida la enfermedad afecta su vida?”, “¿Cuánto cree que durará su enfermedad?”, “¿En qué medida nota síntomas o molestias por su enfermedad?”, “¿Cuánto le preocupa su enfermedad?” y “¿Cuánto le afecta su enfermedad a nivel emocional? (es decir, ¿lo hace sentirse enojado, asustado o deprimido?)".

Para cumplir los objetivos del estudio se utilizaron únicamente cinco de los nueve ítems (1, 2, 5, 6 y 8), y a fin de evaluar la percepción de severidad de enfermedad, entendida como la representación de las consecuencias, temporalidad y síntomas de la enfermedad (identidad) (cf. Horne y Weinman, 2002), se promediaron los ítems 1, 2 y 5, así como los ítems 6 y 8 para evaluar la representación emocional de la enfermedad.

Inventario de Depresión de Beck (IDB) (Beck, Ward, Mendelson, Mock y Erbaugh (1961).

Dicho instrumento consta de 21 reactivos que evalúan la severidad de la sintomatología depresiva. Cada ítem tiene cuatro opciones de respuesta, que van de "nada" a "muy intenso". La puntuación total se calcula mediante la suma de todos los ítems y oscila en un rango que va de 0 a 63 puntos: depresión mínima (0-9), leve (10-16), moderada (17-29) y severa (30-63). El instrumento cuenta con un coeficiente alfa de Cronbach de 0.87 (Jurado et al., 1998).

\section{Procedimiento}

En el caso de los pacientes con ERCT, los investigadores acudieron personalmente a una de las sesiones de hemodiálisis para invitarlos a participar voluntariamente, en tanto que los pacientes con asma fueron abordados en la sala de espera de su clínica antes de entrar a consulta en Neumología. A cada participante se le explicaron los objetivos de la investigación y, en caso de que aceptara participar, se le pidió que leyera y firmara el formato de consentimiento informado, para después hacerle entrega de los instrumentos arriba descritos.

\section{RESULTADOS}

Para el análisis de datos se utilizó el programa estadístico SPSS, v. 20, de Windows. Se llevaron a cabo análisis descriptivos de las variables sociodemográficas, clínicas y de severidad de los síntomas de depresión. Luego se evaluó la normalidad de las variables de representación emocional (ERC: $\mathrm{W}=.924, p=.006$; asma: $\mathrm{W}=.896, p=.001)$, percepción de severidad de la enfermedad (ERC: $\mathrm{W}=.956 ; p=.089$; asma: $\mathrm{W}=.946, p=.046)$, síntomas de depresión (ERC: $\mathrm{W}=.926, p=.007$; asma: $\mathrm{W}=.881, p=.000)$, edad de los participantes (ERC: $\mathrm{W}=.946, p=.036$; asma: $\mathrm{W}=.937$, $p=.022)$ y tiempo de evolución de la enfermedad (ERC: $\mathrm{W}=.907, p=.002$; asma: $\mathrm{W}=.895, p=.001$ ). Dicho análisis se hizo empleando el estadístico Shapiro-Wilk, identificado por Pedrosa, Juarros, Robles, Basteiro y García (2015) como el más adecuado para muestras cercanas a cien participantes cuando se utiliza el programa SPSS. Al no encontrarse normalidad en la mayoría de las variables (con excepción de la de percepción de severidad en ERC), se utilizaron pruebas de comparación y relación no paramétricas.

Se evaluaron las diferencias por sexo empleando tablas de contingencia con $\mathrm{X}^{2} \mathrm{y}$ por ocupación con el estadístico de Kruskal-Wallis, y mediante el estadístico U de Mann-Whitney se compararon la severidad de síntomas de depresión, edad, tiempo de evolución de la enfermedad, percepción de su severidad, representación emocional de la misma y sintomatología de depresión con base en el tipo de enfermedad.

Finalmente, se llevó a cabo un análisis rho de Spearman para identificar la relación entre la percepción de severidad de la enfermedad, la representación emocional y los síntomas de depresión.

Dado que las muestras se seleccionaron de manera intencional, fue necesario compararlas. Como se puede observar en la Tabla 1, se identi- 
ficaron similitudes en ambos grupos al comparar las muestras por el tipo de enfermedad, pero no se observaron diferencias estadísticamente significativas según el sexo $\left(\mathrm{X}^{2}=.458, \mathrm{gl}=1, p=.499\right)$, edad (ERCT: $\mathrm{Me}=38.00$, rango intercuartil $[\mathrm{RI}]=21$, rango promedio $[\mathrm{RP}]=47.49$; asma: $\mathrm{Me}=35.50, \mathrm{RI}=18, \mathrm{RP}=43.51, \mathrm{U}=923.000$, $\mathrm{Z}=-.723, p=.470)$ y tiempo de evolución de la enfermedad (ERCT: $\mathrm{Me}=72.00, \mathrm{RI}=90, \mathrm{RP}=41.81$; asma: $\mathrm{Me}=102.00, \mathrm{RI}=180, \mathrm{RP}=46.35$. $\mathrm{U}=846.500, \mathrm{Z}=-.838, p=.402$ ), por lo que las muestras fueron similares en las variables analizadas no obstante la amplitud de los datos.

En lo que concierne a los datos descriptivos por enfermedad y severidad de los síntomas de depresión, los resultados se muestran en la Tabla 2.

Tabla 2. Categoría de depresión en participantes con ERCT y asma.

\begin{tabular}{|l|c|c|c|c|}
\hline \multirow{2}{*}{$\begin{array}{c}\text { Severidad } \\
\text { de depresión }\end{array}$} & \multicolumn{2}{|c|}{ ERCT $(\mathbf{N}=\mathbf{4 5})$} & \multicolumn{2}{c|}{ ASMA (N = 45) } \\
\cline { 2 - 5 } & Frec. & $\mathbf{\%}$ & Frec. & $\mathbf{\%}$ \\
\hline Mínima & 22 & 48.9 & 21 & 46.7 \\
\hline Leve & 9 & 20.0 & 10 & 22.2 \\
\hline Moderada & 14 & 31.1 & 8 & 17.8 \\
\hline Severa & & & 6 & 13.3 \\
\hline
\end{tabular}

Como se observa en la tabla, los diferentes niveles de severidad de depresión aparecen de manera muy semejante en ambos padecimientos, aunque el porcentaje de personas con síntomas de depresión moderada y severa fue mayor en las personas con asma (13.3\%); en las personas con ERCT ningu- na mostró depresión severa. Al hacer una comparación por enfermedad y severidad de los síntomas de depresión, no se hallaron diferencias estadísticamente significativas a nivel límite $\left(\mathrm{X}^{2}=7.712\right.$, $\mathrm{gl}=3, p=.052$ ).

Ahora bien, al principio se señaló que cada enfermedad tiene diferentes implicaciones debido al tratamiento y a las demandas de la misma, las cuales pueden afectar manera negativamente las actividades realizadas. Debido a ello, se llevó a cabo un análisis de comparación de la severidad de los síntomas de depresión con base en la ocupación de los participantes, sin encontrar diferencias estadísticamente significativas $\left(\mathrm{X}^{2}=.973\right.$, $\mathrm{gl}=3, p=.808$ ), lo que evidencia que dicha severidad es similar en la categoría de ocupación en ambas enfermedades.

El análisis subsecuente consistió en comparar la percepción de severidad de la enfermedad, la representación emocional y los síntomas de depresión. En los resultados se identificaron puntajes por arriba de la media en ambos grupos de pacientes $(>5)$, tanto en el caso de la percepción de severidad, como en la representación emocional; la percepción de severidad fue mayor en los pacientes con ERCT y, por el contrario, la representación emocional lo fue en los pacientes con asma. De igual manera, en los síntomas de depresión en ambos grupos dicho promedio corresponde a una severidad leve de depresión; sin embargo, las diferencias entre grupos no fueron estadísticamente significativas, tal como se muestra en la Tabla 3.

Tabla 3. Comparación entre personas con ERCT y asma en las variables perceptuales y síntomas de depresión.

\begin{tabular}{|c|c|c|c|c|c|c|c|}
\hline Variables de comparación & Enfermedad & Me & RI & $\mathbf{R P}$ & $\mathbf{U}$ & $\mathbf{Z}$ & $p$ \\
\hline \multirow{2}{*}{$\begin{array}{l}\text { Percepción de severidad } \\
\text { (escala de } 0 \text { a } 10 \text { ) }\end{array}$} & ERCT & 5.66 & 2.56 & 47.42 & \multirow{2}{*}{926.000} & \multirow{2}{*}{-.699} & \multirow{2}{*}{.484} \\
\hline & Asma & 5.83 & 4.00 & 43.58 & & & \\
\hline \multirow{2}{*}{$\begin{array}{l}\text { Representación emocional } \\
\text { (escala de } 0 \text { a } 10)\end{array}$} & ERCT & 6.00 & 5.00 & 42.92 & \multirow{2}{*}{896.500} & \multirow{2}{*}{-.939} & \multirow{2}{*}{.347} \\
\hline & Asma & 6.50 & 5.75 & 48.08 & & & \\
\hline \multirow{2}{*}{$\begin{array}{l}\text { Síntomas de depresión } \\
\text { (0 a } 63 \text { puntos) }\end{array}$} & ERCT & 11.00 & 13.00 & 45.91 & \multirow{2}{*}{994.000} & \multirow{2}{*}{-.149} & \multirow{2}{*}{.881} \\
\hline & Asma & 9.50 & 14.00 & 45.09 & & & \\
\hline
\end{tabular}

En términos comparativos, los resultados muestran que los síntomas de depresión no se asocian con el tipo de enfermedad ni con la ocupación de los participantes. Además, se evidencia que la percepción de severidad, la representación emocional y los síntomas de depresión no difieren según el tipo de enfermedad.

Conforme a los resultados hallados, se hizo un análisis de correlación con el propósito de identificar la que había entre las variables relativas a la 
percepción de severidad de la enfermedad, la representación emocional, el tiempo de evolución y los síntomas de depresión. Los resultados se muestran en la Tabla 4.

Tabla 4. Matriz de correlaciones del tipo de enfermedad, percepción de severidad, representación emocional, tiempo de evolución y síntomas de depresión en ERCT y asma.

\begin{tabular}{|c|l|c|c|c|}
\cline { 3 - 5 } \multicolumn{2}{c|}{} & $\begin{array}{c}\text { Representación } \\
\text { emocional }\end{array}$ & $\begin{array}{c}\text { Tiempo } \\
\text { de evolución }\end{array}$ & $\begin{array}{c}\text { Sintomatología } \\
\text { depresiva }\end{array}$ \\
\hline \multirow{3}{*}{ ERCT } & Percepción de severidad & $.717 * *$ & .179 & $.597 * *$ \\
\cline { 2 - 5 } & Representación emocional & & -.027 & $.658 * *$ \\
\cline { 2 - 5 } & Tiempo de evolución & & & .067 \\
\hline \multirow{3}{*}{ Asma } & Percepción de severidad & $.583 * *$ & .083 & $.286^{*}$ \\
\cline { 2 - 5 } & Representación emocional & & $.314 *$ & $.509 * *$ \\
\cline { 2 - 5 } & Tiempo de evolución & & & .090 \\
\hline \multirow{2}{*}{$* p=<.05, * * p$} & & & \\
\end{tabular}

Los resultados correlacionales indican que la percepción de severidad de enfermedad, su representación emocional y los síntomas de depresión se relacionaron positivamente en ambos padecimientos; es decir, a mayor percepción de severidad de enfermedad y mayor representación emocional de la misma, más elevados fueron los síntomas de depresión, y viceversa.

Por el contrario, el tiempo de evolución de la enfermedad no se asoció con la percepción de su severidad, la representación emocional y los síntomas de depresión en pacientes con ERCT, y tampoco entre el tiempo de evolución, la percepción de severidad de la enfermedad y los síntomas de depresión en pacientes con asma; sin embargo, se halló una relación entre el tiempo de evolución y la representación emocional.

Los resultados sugieren que los síntomas de depresión se asocian con las variables perceptuales de severidad de la enfermedad y la representación emocional, y no con el tiempo que se lleve enfermo. En este sentido, se evidencia la importancia de las variables psicológicas de representación de la enfermedad y su relación con los síntomas de depresión, no así en los casos de las variables sociodemográficas y clínicas, como el tiempo de evolución, el tipo de enfermedad y la ocupación.

Finalmente, es necesario subrayar que, en función de la severidad de enfermedad en términos biomédicos, no hubo diferencias en las variables perceptuales y de sintomatología depresiva, lo que apunta a que el impacto emocional de la enfermedad se asocia en mayor medida a la forma de percibir o representar el padecimiento.

\section{DISCUSIÓN}

De acuerdo con los resultados obtenidos, es posible concluir que los síntomas de depresión en enfermos crónicos es uno de los principales problemas de carácter psicológico (Clarke y Currie, 2009; Pagoto, 2011). En el presente estudio se identificó que más de la mitad de los pacientes en ambas muestras presentaba síntomas de depresión de leve a severa, y que una tercera parte mostró síntomas de depresión de moderados a severos, datos que se corresponden con lo descrito en la literatura en diferentes partes del mundo sobre los pacientes con ERCT (Esquivel et al., 2009; McKercher et al., 2013; Moreno et al., 2004) y asma (Coban y Aydemir, 2014; Di Marco et al., 2010; Ryu et al., 2010), mismos que se han considerado con relevancia clínica (Kellerman, Christensen, Baldwin y Lawton, 2010).

En cuanto a la percepción de la enfermedad y los síntomas de depresión, los datos se agrupan en la misma línea; por ejemplo, en pacientes con ERCT se identifica que la severidad de la enfermedad, determinada con base en criterios médicos, no se relaciona con los síntomas de depresión; por el contrario, la percepción negativa de la enfermedad impacta y se relaciona directamente con los síntomas de depresión (Guzman y Nicassio, 2003; Ibrahim et al., 2011; Lin et al., 2013; Rees et al., 2018). Estos datos corresponden de igual manera a los pacientes con asma, en quienes se ha visto que el percibir mayores consecuencias y el mostrar una mayor percepción de severidad de la enfermedad se asocian directamente con indicado- 
res de depresión (Bahçecioğlu y Çevikakyl, 2014; Lugo et al., 2014; Thuné-Boyle et al., 2006).

Ahora bien, es necesario reiterar que los datos de las investigaciones mostradas y contrastadas se discuten a la luz de la representación de la enfermedad, el principal supuesto del MSCRE (Diefenbach y Leventhal, 1996), que postula que la forma de percibirla será diferente en cada persona; es, pues, individualizada y no necesariamente se asocia con los criterios médicos (Diefenbach y Leventhal, 1996; Leventhal, Meyer y Nerenz, 1980). Por lo tanto, las repercusiones emocionales derivadas de sufrir una enfermedad crónica no dependerán de la severidad de la misma basada en la categorización médica, sino de la forma en que se le perciba.

En el MSCRE se considera que cada persona está en un proceso continuo de adaptación, ya que entenderá su enfermedad sobre la base de su representación. Dicha representación se modifica a través del tiempo, por lo que influirá de manera constante y cambiante en las conductas de afrontamiento, así como en las repercusiones emocionales (Diefenbach y Leventhal, 1996). Por consiguiente, sería inadecuado considerar que un grupo de personas que padezcan una enfermedad crónica se encontrarán emocionalmente más comprometidas que otro grupo al calificarla como más grave. Por ello, cobra sentido tomar en cuenta que las diferencias entre pacientes con ERCT y asma respecto a su estado emocional no sean muy amplias, y que de hecho puede haber pacientes con niveles de depresión más severos en el grupo en que se considera que el padecimiento no es tan grave.

Tomar como base la percepción de enfermedad resulta de gran relevancia en la investigación y en la intervención clínica; al menos así lo aseguran Leventhal, Breland, Mora y Leventhal (2010), Farmer (2012) y Kucukarslan (2016), quienes ponen de manifiesto que en el contexto clínico se trabaja escasamente con las representaciones y las formas de afrontamiento ante la enfermedad. Por ello, el trabajo relativo a las creencias - particularmente desde el MSCRE- es un gran apoyo ya que funge como un modelo psicológico centrado en el paciente, en cuanto que explica y aborda las perspectivas, formas de afrontar la enfermedad y de clarificar las repercusiones emocionales en diversos padecimientos crónicos (Barton, Clarke, Sulaiman y Abramson, 2003; Farmer, 2012; Horne et al., 2007?; Kucukarslan, 2016; Leventhal et al., 2010).

Una de las principales limitaciones del presente estudio es el tipo de muestreo empleado. Los participantes se seleccionaron según su disponibilidad y facilidad de acceso. Por consiguiente, sería útil incluir otro de tipo de pacientes con enfermedades distintas a las del presente análisis. Otra limitación fue que no se incluyó la variable relativa a la severidad de la enfermedad (intermitente, leve, moderada y severa) en los pacientes con asma en virtud de que se podrían encontrar resultados contrastantes respecto a los síntomas de depresión y a la percepción de enfermedad.

Una limitación adicional fue el empleo de ítems únicos en la evaluación de la representación de enfermedad (B-IPQ). En los estudios de revisión más actuales sobre la evaluación de la representación de enfermedad mediante instrumentos se ha sugerido que el B-IPQ tiene mejores resultados cuando se le aplica en la clínica y no tanto en la investigación, recomendándose para esta última instancia el empleo del IPQ-R (Broadbent et al., 2015). Aunado a ello, el uso de ítems únicos ha sido objeto de diversos debates por los problemas de imprecisión en las mediciones (Fuch y Diamantopolus, 2009); sin embargo, a modo de defensa, es relevante señalar que hay pruebas que demuestran que los ítems únicos para evaluar diversas variables son igualmente útiles que los ítems múltiples (Bergkvist y Rossiter, 2007). Además, los ítems únicos se pueden utilizar si la muestra a evaluar es limitada y heterogénea (Fuch y Diamantopolus, 2009), como fue el caso presente.

Para futuras investigaciones se propone aumentar el número de la muestra e incluir otros tipos de enfermedades crónicas para así llevar a cabo diversas comparaciones, evaluar otro tipo de variables psicosociales y emplear herramientas de evaluación más amplias. 


\section{REFERENCIAS}

Bahçecioğlu, G. y Çevikakyl, R. (2014). Determination of effect on asthma control of illness perception of asthma patients. Acta Medica Mediterránea, 30, 591-600.

Barley, E. y Lawson, V. (2016). Health psychology: supporting the self-management of long-term conditions. British Journal of Nursing, 25(20), 1102-1107.

Barton, C., Clarke, D., Sulaiman, N. y Abramson, M. (2003). Coping as a mediator of psychosocial impediments to optimal management and control of asthma. Respiratory Medicine, 97(7), 747-761. doi: 10.1016/S0954-6111(03)00029-5.

Beck, A.T., Ward, C.H., Mendelson, M., Mock, J. y Erbaugh, J. (1961). An inventory for measuring depression. Archives of General Psychiatry, 4, 561-571.

Bergkvist, L. y Rossiter, J.R. (2007). The predictive validity of multiple-item versus single-item measures of the same constructs. Journal of Marketing Research, 44(2), 175-184.

Bravo G., M.C. (2014). Generalidades psicológicas de los padecimientos crónicodegenerativos. En L. Reynoso y A. L. Becerra (Eds): Teoría y práctica de la medicina conductual (pp. 43-64). México: Qartuppi.

Broadbent, E., Petrie, K., Main, J. y Weinman, J. (2006). The Brief Illness Perception Questionnaire. Journal of Psychosomatic Research, 60, 631-637. doi: 10.1016/j.jpsychores.2005.10.020.

Broadbent, E., Wilkes, C., Koschwanez, H., Weinman, J., Norton, S. y Petrie, K.J. (2015). A systematic review and meta-analysis of the Brief Illness Perception Questionnaire. Psychology y Health, 30(11), 1361-1385. doi: 10.1080/08870446.2015.1070851.

Cameron, P. y Moss-Morris, R. (2004). Illness-related cognition and behavior. En A. A., Kaptein y J. Weinman (Eds.): Health Psychology (pp. 84-110), Oxford, UK: BPS Blackwell.

Choo, E.M., Hoyte, F.C.L. y Katial, R.K. (2014). Adult and pediatric asthma. En P. K. Vedanthan, H. Nelson, S. H. Agashe, P. A. Mahesh y R. Katial (Eds.): Textbook of allergy for the clinician (pp. 135-142). Boca Raton, FLO: CRC Press Taylor y Francis Group.

Christensen, A. y Ehlers, S. (2002). Psychological factors in end-stage renal disease: An emerging context for behavioral medicine research. Journal of Consulting and Clinical Psychology, 70(3), 712-724. doi: 10.1037/0022-006X.70.3.712.

Ciprandi, G., Schiavetti, I., Rindone, E. y Ricciardolo, F.L. (2015). The impact of anxiety and depression on outpatients with asthma. Annals of Allergy, Asthma \& Immunology, 115(5), 408-414.

Clarke, D.M. y Currie, K.C. (2009). Depression, anxiety and their relationship with chronic diseases: a review of the epidemiology, risk and treatment evidence. Medical Journal of Australia, 190(7), S54. PMID: 19351294.

Coban, H. y Aydemir, Y. (2014). The relationship between allergy and asthma control, quality of life, and emotional status in patients with asthma: a cross-sectional study. Allergy, Asthma \& Clinical Immunology, 10(1), 67. doi: 10.1186/s13223014-0067-4.

Cukor, D., Coplan, J., Brown, C., Friedman, S., Cromwell-Smith, A., Peterson, R.A. y Kimmel, P.L. (2007). Depression and anxiety in urban hemodialysis patients. Clinical Journal of the American Society of Nephrology, 2(3), 484-490. doi: 10.2215/CJN.00040107.

Deshmukh, V., Toelle, B., Usherwood, T., O’Grady, B. y Jenkins, C. (2008). The association of comorbid anxiety and depression with asthma-related quality of life and symptom perception in adults. Respirology, 13, 695-702. doi: 10.1111/j.14401843.2008.01310.x.

Diefenbach, M.A. y Leventhal, H. (1996). The common-sense model of illness representation: Theoretical and practical considerations. Journal of Social Distress and the Homeless, 5(1), 11-38. doi: 10.1007/BF02090456.

DiMatteo, M.R. (2004). Variations in patients' adherence to medical recommendations: A quantitative review of 50 years of research. Medical Care, 42(3), 200-209. doi: 10.1097/01.mlr.0000114908.90348.f9.

Di Marco, F., Verga, M., Santus, P., Giovannelli, F., Busatto, P., Neri, M., Girbino, G., Bonini, S. y Centanni, S. (2010). Close correlation between anxiety, depression, and asthma control. Respiratory Medicine, 104, 22-28. doi: 10.1016/j.rmed.2009.08.005.

Esquivel M., C.G., Prieto F., J.G., López R., J., Ortega C., R., Martínez M., J.A. y Velasco R., V. M. (2009). Calidad de vida y depresión en pacientes con insuficiencia renal crónica terminal en hemodiálisis. Medicina Interna de México, $25(4), 443-449$.

Espinosa, F., Parra, M., Segura, N., Toledo, D., Ménez, D., Sosa E. y Torres, A. (2006). Ansiedad y depresión en asmáticos adultos en comparación con sujetos sanos. Revista Alergia México, 53(6), 201-206.

Farmer, K.C. (2012). Leventhal's common-sense model and medication adherence. Research in Social and Administrative Pharmacy, 8, 355-356. doi: 10.1016/j.sapharm.2012.05.013.

Foster, J.M., Lavoide, K.L. y Boulet, L.P. (2011). Treatment adherence and psychosocial factors in severe asthma. En K. F. Chung, E. H. Bel y S. E. Wenzel (Eds.): European Respiratory Monograph: Dificult to treat severe asthma, 51 (pp. 28-49). European Respiratory Society. doi: 10.1183/1025448x.erm5110.

Fuchs, C. y Diamantopoulos, A. (2009). Using single-item measures for construct measurement in management research: conceptual issues and application guidelines. Die Betriebswirtschaft, 69(2), 195. http://www.dbwnet.de/. 
Gerontoukou, E.I., Michaelidoy, S., Rekleiti, M., Saridi, M. y Souliotis, K. (2015). Investigation of anxiety and depression in patients with chronic diseases. Health Psychology Research, 3(2), 2015-2123. doi: 10.4081/hpr.2015.2123.

Global Initiative for Asthma (2016). Global strategy for asthma management and prevention. Recuperado de www.ginasthma.org.

Goh, Z.S. y Griva, K. (2018). Anxiety and depression in patients with end-stage renal disease: impact and management challenges-a narrative review. International Journal of Nephrology and Renovascular Disease, 11, 93-102. doi: 10.2147/IJNRD. S126615.

González, L., Sánchez, S., Morales, L., Ostrosky, F., Alberú, J., García, G., Marino, Ll. y McClintock, S. (2011). Assesment of emotional distress in chronic kidney disease patients and kidney transplant recipients. Revista de Investigación Clínica, 63(6), 558-563. PMID: 23650668.

Grau A., J.A. (2016). Factores psicosociales y enfermedades crónicas: el gran desafío. Revista Latinoamericana de Medicina Conductual, 6(2), 103-112.

Guzman, S.J. y Nicassio, P.M. (2003). The contribution of negative and positive illness schemas to depression in patients with end-stage renal disease. Journal of Behavioral Medicine, 26(6), 517-534. doi: 10.1023/A:1026249702054.

Halm, E., Mora, P. y Leventhal, H. (2006). No symptoms, no asthma. The acute episodic disease beliefs is associated with poor self-management among inner-city adults with persistent asthma. Chest, 129, 573-580. doi: 10.1378/chest.129.3.573.

Hernández S., R., Fernández C., C. y Baptista L., P. (2014). Metodología de la investigación. México: McGraw-Hill.

Hill-Briggs. F. (2003). Problem solving in diabetes self-management: A model of chronic illness self-management behavior. Annals Behavior Medicine, 25(3), 182-193. doi: 10.1207/S15324796ABM2503_04.

Horne, R., Price, D., Cleland, J., Costa, R., Covey, D., Gruffydd-Jones, K., Haughney, J., Hoegh, S., Kaplan, A., Langhammer, A., Østrem, A., Thomas, M., van der Molen, T., Virchow, J. C. y Williams, S. (2007). Can asthma control be improved by understanding the patient's perspective? BMC Pulmonary Medicine, 7(1), 1-11. doi: 10.1186/1471-2466-7-8.

Horne, R. y Weinman, J. (2002). Self-regulation and self-management in asthma: Exploring the role of illness perceptions and treatment beliefs in explaining non-adherence to preventer medication. Psychology \& Health, 17(1), 17-32. doi: 10.1080/08870440290001502.

Ibrahim, N., Chiew-Tong, N.K. y Desa, A. (2011). Illness perception and depression with end-stage renal disease on chronic hemodialysis. The Social Sciences, 6(3),221-226. doi: 10.3923/sscience.2011.221.226.

Jansen, D., Heijmans, M., Rijken, M., Spreeuwenberg, P., Grootendorst, D., Dekker, F., Boeschoten, E., Kaptein, A. y Groenewegen, P. (2013). Illness perceptions and treatment perceptions of patients with chronic kidney disease: different phases, different perceptions? British Journal of Health Psychology, 18(2), 244-262. doi:10.1111/bjhp.12002.

Jansen, D., Rijken, M., Heijmans, M., Kaptein, A. y Groenewegen, P. (2012). Psychological and social aspects of living with chronic kidney disease. En S. Manisha (Ed.): Chronic kidney disease and renal transplantation (pp 47-74). Amsterdam: InTech. doi: 10.5772/25992.

Jurado, S., Villegas, M., Méndez, L., Rodríguez, F., Loperena, V. y Varela, R. (1998). La estandarización del Inventario de Depresión de Beck para los residentes de la Ciudad de México. Salud Mental, 21, 26-31. http://biblat.unam.mx/es/revista/ salud-mental/.

Kaptein, A.A., Hughes, B.M., Scharloo, M., Fischer, M.J., Snoei, L., Weinman, J. y Rabe, K.F. (2008). Illness perceptions about asthma are determinants of outcome. Journal of Asthma, 45(6), 459-464. doi: 10.1080/02770900802040043.

Kaptein, A., Klok, T., Moss-Morris, R. y Brand, P. (2010). Illness perceptions: impact on self-management and control in asthma. Current Opinion in Allergy and Clinical Immunology, 10, 194-199. doi: 10.1097/ACI.0b013e32833950c1.

Katon, W.J. (2011). Epidemiology and treatment of depression in patients with chronic medical illness. Dialogues in Clinical Neuroscience, 13(1), 7.

Kellerman, Q.D., Christensen, A.J., Baldwin, A.S. y Lawton, W.J. (2010). Association between depressive symptoms and mortality risk in chronic kidney disease. Health Psychology, 29(6), 594. doi: 10.1037/a0021235.

Kucukarslan, S.N. (2016). Using the common sense model in daily clinical practice for improving medication adherence. Journal of Clinical Outcomes Management, 23(5), 227-230. www.jcomjournal.com.

Larsen, P. (2009). Chronicity. En P. D. Larsen e I. M. Lubkin (Eds.): Chronic illness: Impact and intervention (7th ed.) (pp 3-24). Burlington, MA: Jones and Bartlett Publishers, LLC.

Leventhal, H., Breland, J.Y., Mora, H. y Leventhal, E. (2010). Lay representations of illness and treatment: A framework for action. En A. Steptoe (Ed.): Handbook of Behavioral Medicine (pp. 137-154). New York: Springer Science-Business Media. doi: 10.1007/978-0-387-09488-5_11.

Leventhal, H., Meyer, D. y Nerenz, D. (1980). The common sense representation of illness danger. En: S. Rachman (Ed.): Contributions to medical psychology (v. II, pp. 9-33). London: Pergamon Press Ltd.

Lin, C.C., Chen, M.C., Hsieh, H.F. y Chang, S.C. (2013). Illness representations and coping processes of Taiwanese patients with early-stage chronic kidney disease. Journal of Nursing Research, 21(2), 120-128. doi: 10.1097/jnr.0b013e3182921fb8.

Livneh, H., Lott, S. y Antonak, R. (2004). Patterns of psychosocial adaptation to chronic illness and disability: A cluster analytic approach. Psychology, Health \& Medicine, 9(4), 411-429. doi: 10.1080/1354850042000267030. 
Livneh, H. y Antonak, R. (2005). Psychosocial adaptation to chronic illness and disability: A primer for counselors. Journal of Counseling \& Development, 83, 12-20. doi: 10.1002/j.1556-6678.2005.tb00575.x.

Lugo, I., Reynoso, L. y Fernández V., M. (2014). Percepción de enfermedad, depresión, ansiedad y control del asma: una primera aproximación. Neumología y Cirugía de Tórax, 73(2), 114-121.

McKercher, Ch., Venn, A., Blizzard, L., Nelson, M., Palmer, A., Ashby, M., Scott, J. y Jose, M. (2013). Psychological factors in adults with chronic kidney disease: characteristics of pilot participants in the Tasmanian Chronic Kidney Disease study. BMC Nephrology, 14(83), 14-28. doi: 10.1186/1471-2369-14-83.

Méndez, I., Namihira, D., Moreno, L. y Sosa, C. (2001). El protocolo de investigación. Lineamientos para su elaboración y análisis. México: Trillas.

Mora, P.A. y McAndrew, L.M. (2013). Common-sense model of self-regulation. En M.D. Gellman y J.R. Turner (Eds.): Encyclopedia of Behavioral Medicine (pp. 460-467). New York: Springer. doi: 10.1007/978-1-4419-1005-9.

Moreno N., E., Arenas J., M., Porta B., E., Escalant C., L., Cantó G., M., Castell G., G., Serrano G., F., Samper L., J., Millán M., D. y Cases I., J. M. (2004). Estudio de la prevalencia de trastornos ansiosos y depresivos en pacientes en hemodiálisis. Revista de la Sociedad Española de Enfermería Nefrológica, 7(4), 17-25. doi: 10.4321/S2254-28842015000200006.

Moss-Morris, R., Weinman, J., Petrie, K., Horne, R., Cameron, L. y Buick, D. (2002). The Revised Illness Perception Questionnaire (IPQ-R). Psychology \& Health, 17(1), 1-16. doi: 10.1080/08870440290001494.

Mucsi, I. (2008). Health-related quality of life in chronic kidney disease patients. Primary Psychiatry, 15(1), 46-51.

Opolski, M. y Wilson, I. (2005). Asthma and depression: a pragmatic review of the literature and recommendations for future research. Clinical Practice and Epidemiology in Mental Health, 1(18), 1-7. doi: 10.1186/1745-0179-1-18.

Pagoto, S. (2011). Psychological co-morbidities of physical illness: A behavioral medicine perspective. New York: Springer. doi: 10.1007/978-1-4419-0029-6.

Pedrosa, I., Juarros B., J., Robles F., A., Basteiro, J. y García C., E. (2015). Pruebas de bondad de ajuste en distribuciones simétricas, ¿qué estadístico utilizar? Universitas Psychologica, 14(1), 15-24. doi: 10.11144/Javeriana.upsy14-1.pbad.

Petrie, K., Jago, L. y Devcich, D. (2007). The role of illness perceptions in patients with medical conditions. Current Opinion in Psychiatry, 20, 163-167. doi: 10.1097/YCO.0b013e328014a871.

Rees, J., Chilcot, J., Donnellan, W. y Soulsby, L. (2018). Exploring the nature of illness perceptions in people with end-stage kidney disease. Journal of Renal Care, 44(1), 19-29. doi: 10.1111/jorc.12225.

Ritz, T., Meuret, A.E., Trueba, A.F., Fritzsche, A. y von Leupoldt, A. (2013). Psychosocial factors and behavioral medicine interventions in asthma. Journal of Consulting and Clinical Psychology, 81(2), 231. doi: 10.1037/a0030187.

Ryu, Y.J., Chun, E.M., Lee, J.H. y Chang, J. H. (2010). Prevalence of depression and anxiety in outpatients with chronic airway lung disease. The Korean Journal of Internal Medicine, 25(1), 51-57. doi: 10.3904/kjim.2010.25.1.51.

Skorecki, K., Green, J. y Breenner, B. (2001). Insuficiencia renal crónica. En E. Braunwald., A. Favci., D. Kasper, S. Hauser, D. Longo y J. Jameson (Comp.): Principios de Medicina Interna (v. II, pp. 1815-1839). México: McGraw-Hill.

Strine, T.W., Mokdad, A.H., Balluz, L.S., Berry, J.T. y Gonzalez, O. (2008). Impact of depression and anxiety on quality of life, health behaviors, and asthma control among adults in the United States with asthma, 2006. Journal of Asthma, 45(2), 123133. doi: 10.1080/02770900701840238.

Thuné-Boyle, I.C., Myers, L.B. y Newman, S.P. (2006). The role of illness beliefs, treatment beliefs, and perceived severity of symptoms in explaining distress in cancer patients during chemotherapy treatment. Behavioral Medicine, 32(1), 19-29. doi: 10.3200/BMED.32.1.19-29.

Unni, E. y Shiyanbola, O.O. (2016). Clustering medication adherence behavior based on beliefs in medicines and illness perceptions in patients taking asthma maintenance medications. Current Medical Research and Opinion, 32(1), 113-121. doi: 10.1185/03007995.2015.1105204.

Van Lieshout, R. y MacQueen, G. (2008). Psychological factors in asthma. Allergy, Asthma and Clinical Immunology, 4(1), 12-28. doi: 10.1186/1710-1492-4-1-12.

Walker, H. y Chen, E. (2010). The impact of family asthma management on biology: a longitudinal investigation of youth with asthma. Journal of Behavioral Medicine, 33, 326-334. doi: 10.1007/s10865-010-9258-8.

Yorke, J., Fleming, S. y Shuldham, C. (2007). Psychological interventions for adults with asthma: A systematic review. Respiratory Medicine, 101, 1-14. doi: 10.1016/j.rmed.2006.04.003. 